\title{
Joint Relay and Jammer Selection for Secure Two-Way Relay Networks
}

\author{
Jingchao Chen, Rongqing Zhang, Lingyang Song, Zhu Han ${ }^{\dagger}$, and Bingli Jiao \\ School of Electrical Engineering and Computer Science, Peking University, Beijing, China. \\ ${ }^{\dagger}$ Electrical and Computer Engineering Department, University of Houston, USA.
}

\begin{abstract}
In this paper, we investigate joint relay and jammer selection in two-way cooperative networks, consisting of two sources, a number of intermediate nodes, and one eavesdropper, with the constraints of physical layer security. Specifically, the proposed algorithms select two or three intermediate nodes to enhance security against the malicious eavesdropper. The first selected node operates in the conventional relay mode and assists the sources to deliver their data to the corresponding destinations using an amplify-and-forward protocol. The second and third nodes are used in different communication phases as jammers in order to create intentional interference upon the eavesdropper node. Firstly, we find that in a topology where the intermediate nodes are randomly and sparsely distributed, the proposed schemes with cooperative jamming outperform the conventional nonjamming schemes within a certain transmitted power regime. We also find that, in the scenario in which the intermediate nodes gather as a close cluster, the jamming schemes may be less effective than their non-jamming counterparts. Therefore, we introduce a hybrid scheme to switch between jamming and non-jamming modes. Simulation results validate our theoretical analysis and show that the hybrid switching scheme further improves the secrecy rate.
\end{abstract}

This work is partially supported by US NSF CNS-0910401, CNS-0905556, and CNS-0953377. 


\section{INTRODUCTION}

Traditionally security in wireless networks has been mainly focused on higher layers using cryptographic methods [1]. Pioneered by Aaron Wyner's work [2], which introduced the wiretap channel and established fundamental results of creating perfectly secure communications without relying on private keys, physical-layer-based security has drawn increasing attention recently. The basic idea of physical layer security is to exploit the physical characteristics of the wireless channel to provide secure communications. The security is quantified by the secrecy capacity, which is defined as the maximum rate of reliable information sent from the source to the intended destination in the presence of eavesdroppers. Wyner showed that when the eavesdropper channel is a degraded version of the main channel, the source and the destination can exchange secure messages at a non-zero rate. The following research work [3] studied the secrecy capacity of the Gaussian wiretap channel, and [4] extended Wyner's approach to the transmission of confidential messages over the broadcast channels. Very recently, physical layer security have been generalized to investigate wireless fading channels [5]-[8], and various multiple access scenarios [9]-[12].

Note the fact that if the source-wiretapper channel is stronger than the source-destination channel, the perfect secrecy rate will be zero [4]. Some work [13]-[24] has been proposed to overcome this limitation with the help of relay cooperation by cooperative relaying [13][14], and cooperative jamming [15]-[17]. For instance, in [13] and [14], the authors proposed effective decode-and-forward (DF) and amplify-and-forward (AF) based cooperative relaying protocols for physical layer security, respectively. Cooperative jamming is another approach to improve the secrecy rate by interfering the eavesdropper with codewords independent of the source messages. In Yener and Tekin's work [15], a scheme termed collaborative secrecy was proposed, in which a non-transmitting user was selected to help increase the secrecy capacity for a transmitting user by effectively "jamming" the eavesdropper. Following similar idea as [15], they first proposed cooperative jamming in [16] and [17] in order to increase achievable rates in the scenarios where general gaussian multiple access wire-tap channel and two-way wire-tap channel were assumed, respectively. The authors of [18] and [19] investigated the effects of user cooperation on the secrecy of broadcast channels by considering a cooperative relay broadcast channel, and showed that user cooperation can increase the achievable secrecy region. The study of communicating through unauthenticated intermediate relays between a source-destination pair started from Yenner and He's work [20]-[22]. The relay channel with 
confidential messages was also investigated in [23]-[24], where the untrusted relay node acts both as an eavesdropper and a conventional assistant relay.

Two-way communication is a common scenario in which two nodes transmit information to each other simultaneously. Recently, the two-way relay channel [25]-[29] has attracted lots of interest from both academic and industrial communities due to its bandwidth efficiency and potential application to cellular networks and peer-to-peer networks. In [25] and [26], both AF and DF protocols for one-way relay channels were extended to general full-duplex discrete two-way relay channel and half-duplex Gaussian two-way relay channel, respectively. In [27], network and channel coding were used in two-way relay channel to increase the sum-rate of two sources. The work in [28] introduced a two-way memoryless system with relays in which the signal transmitted by the relay was obtained by applying an instantaneous relay function to the previously received signal in order to optimize the symbol error rate performance. As for the secure communications, in [29], Yener and He investigated the role of feedback in secrecy for two-way networks, and proved that the loss in secrecy rate when ignoring the feedback is very limited in a scenario with half-duplex Gaussian two-way relay channels and an eavesdropper.

It is well known that, in a cooperative communication network, proper relay/jammer selection can have a significant impact on the performance of the whole system. Several relay selection techniques [30]-[32] have been explored by far. The authors in [30] proposed a non-jamming relay selection scheme for two-way networks with multiple AF relays in an environment without eavesdroppers, which maximized the worse received signal-to-noise ratio (SNR) of the two end users. In [31], several relay selection techniques were proposed in one-way cooperative networks with secrecy constraints. In [32], the authors investigated some relay selection techniques in a two-hop DF cooperative communication system with no central processing unit to optimally select the relay. Although cooperative networks have received much attention by far, the physical layer security issues with secrecy constraints in two-way schemes have not yet been well investigated.

To this end, in this paper, we propose a scheme that can implement information exchange in the physical layer against eavesdroppers for two-way cooperative networks, consisting of two sources, a number of intermediate nodes, and one eavesdropper, with the constraints for physical layer security. Unlike [30], in which the relay selection is operated in an environment with no security requirement, our work takes into account the secrecy constraints. In contrast to [31], where many relay selections based on the DF strategy for one-way cooperative 
wireless networks were proposed and a safe broadcast phase was assumed, the problem we consider here involves a non-security broadcast phase, and the information is transferred bidirectionally.

Specifically, a node is selected from an intermediate node set to operate at a conventional relay mode, and then uses an AF strategy in order to assist the sources to deliver data to the corresponding destination. Meanwhile, another two intermediate nodes that perform as jammers are selected to transmit artificial interference in order to degrade the eavesdropper links in the first and second phases of signal transmissions, respectively. We assume that both destinations cannot mitigate artificial interference, and thus, the jamming will also degrade the desired information channels. The principal question here is how to select the relay and the jamming nodes in order to increase information security, and meanwhile protect the source message against eavesdroppers. Several selection algorithms are proposed, aiming at promoting the assistance to the sources as well as the interference to the eavesdropper.

The theoretical analysis and simulation results reveal that the proposed jamming schemes can improve the secrecy rate of the system by a large scale, but only within a certain transmitted power range. In some particular scenarios, the proposed schemes become less efficient than the conventional ones. We then propose a hybrid scheme with an intelligent switching mechanism between jamming and non-jamming modes to solve this problem.

The rest of this paper is organized as follows. In Section II, we describe the system model, and formulate the problem under consideration. Section III presents the proposed selection techniques, and introduces their hybrid implementations. In Section IV, we provide both quantitative analysis and qualitative discussions of different selection schemes in some typical configurations. Numerical results are shown in Section V, and in Section VI, we draw the main conclusions.

\section{System Model ANd Problem Formulation}

\section{A. System Model}

We assume a network configuration consisting of two sources $S_{1}$ and $S_{2}$, one eavesdropper $E$, and an intermediate node set $S_{i n}=\{1,2, \ldots, K\}$ with $K$ nodes. In Fig. 1 it schematically shows the system model. As the intermediate nodes cannot transmit and receive simultaneously (half duplex constraint), the communication process is performed into two phases. During the broadcasting phase, $S_{1}$ and $S_{2}$ transmit their data to the intermediate nodes. In addition, according to the security protocol, one node $J_{1}$ is selected from $S_{i n}$ to operate as 


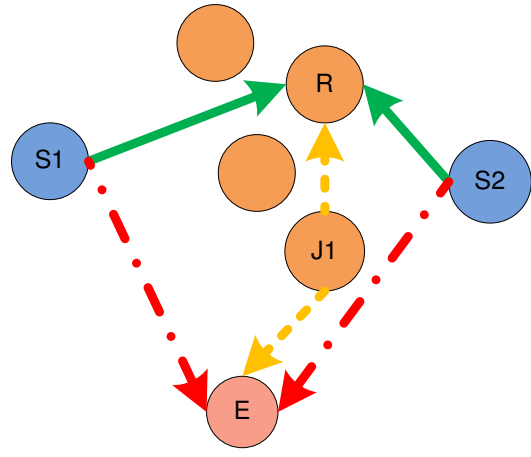

1st phase

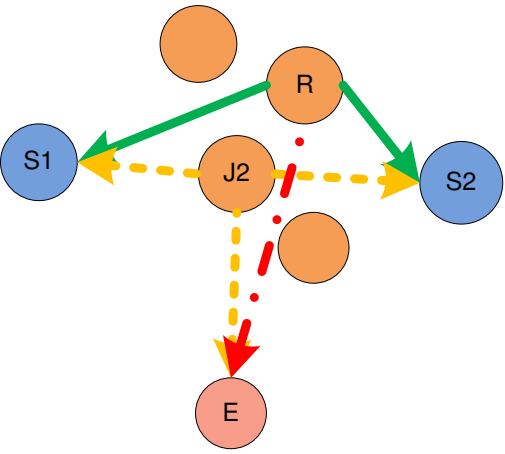

2nd phase

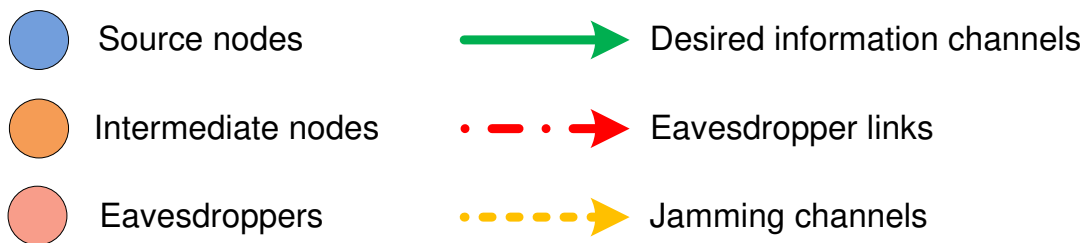

Fig. 1. System model, where the eavesdropper node is able to receive signals from both $S_{1}$ and $S_{2}$.

a "jammer" and transmit intentional interference to degrade the eavesdropper links in this phase. Since the jamming signal is unknown at the rest nodes of $S_{i n}$, the interference will also degrade the performance of the relay links, as shown in Fig. 1. In the second phase, according to the security protocol, an intermediate node $R$ is selected to operate as a conventional relay and forwards the source messages to the corresponding destinations. A second jammer $J_{2}$ is selected from $S_{i n}$, for the same reason as that for $J_{1}$. Note that the destinations $S_{1}$ and $S_{2}$ are not able to mitigate the artificial interference from the jamming node, either.

In both two phases, a slow, flat, and block Rayleigh fading environment is assumed, i.e., the channel remains static for one coherence interval and changes independently in different coherence intervals with a variance $\sigma_{i, j}^{2}=d_{i, j}^{-\beta}$, where $d_{i, j}$ denotes the Euclidean distance between node $i$ and node $j$, and $\beta$ represents the path-loss exponent. The channel between node $i$ and node $j$ is denoted as $h_{i, j}$, which is modeled as a zero-mean, independent, circularlysymmetric complex Gaussian random variable with variance $\sigma_{i, j}^{2}$. Furthermore, additive white Gaussian noise (AWGN) with zero mean and unit variance is assumed. Let $P_{S}, P_{R}$ and $P_{J}$ denote the transmitted power for the source nodes, the relay node and the jamming nodes, respectively. In order to protect the destinations from severe artificial interference, the jamming nodes transmit with a lower power than the relay nodes [31], and thus their 
transmitted power can be defined as $P_{J}=P_{R} / L$, where $L \gg 1$ denotes the power ratio of the relay to the jammer.

In the first phase, the two sources send information symbols $s_{1}$ and $s_{2}$, respectively, which are mapped to a PSK set. The intermediate node $R$ and eavesdropper $E$ thus receive

$$
\begin{aligned}
r & =\sqrt{P_{S}} h_{S_{1}, R} s_{1}+\sqrt{P_{S}} h_{S_{2}, R} s_{2}+\sqrt{P_{J}} h_{J_{1}, R} j_{1}+v_{R}, \\
e_{1} & =\sqrt{P_{S}} h_{S_{1}, E} s_{1}+\sqrt{P_{S}} h_{S_{2}, E} s_{2}+\sqrt{P_{J}} h_{J_{1}, E} j_{1}+v_{E},
\end{aligned}
$$

where $v_{R}$ and $v_{E}$ denote the noise at $R$ and $E$, respectively.

In the second phase, the node $R$ is selected to amplify its received signal and forward it to $S_{1}$ and $S_{2}$, i.e., $R$ broadcasts

$$
t=\alpha \sqrt{P_{R}} r
$$

where $\alpha=\sqrt{\frac{1}{1+\left|h_{S_{1}, R}\right|^{2} P_{S}+\left|h_{S 2, R}\right|^{2} P_{S}+\left|h_{J_{1}, R}\right|^{2} P_{J}}}$.

Since the destination $S_{i}$ knows $s_{i}$ (for $i=1,2$ ), it can cancel the self-interference. Therefore, $S_{1}, S_{2}$, and the eavesdropper $E$ get

$$
\begin{aligned}
x_{1}= & \alpha \sqrt{P_{R}} \sqrt{P_{S}} h_{S_{2}, R} h_{R, S_{1}} s_{2}+\alpha \sqrt{P_{R}} \sqrt{P_{J}} h_{J_{1}, R} h_{R, S_{1}} j_{1} \\
& +\sqrt{P_{J}} h_{J_{2}, S_{1}} j_{2}+\alpha \sqrt{P_{R}} h_{R, S_{1}} v_{R}+w_{1}, \\
x_{2}= & \alpha \sqrt{P_{R}} \sqrt{P_{S}} h_{S_{1}, R} h_{R, S_{2}} s_{1}+\alpha \sqrt{P_{R}} \sqrt{P_{J}} h_{J_{1}, R} h_{R, S_{2}} j_{1} \\
& +\sqrt{P_{J}} h_{J_{2}, S_{2}} j_{2}+\alpha \sqrt{P_{R}} h_{R, S_{2}} v_{R}+w_{2}, \\
e_{2}= & \alpha \sqrt{P_{R}} \sqrt{P_{S}}\left(h_{S_{1}, R} s_{1}+h_{S_{2}, R} s_{2}\right) h_{R, E}+\alpha \sqrt{P_{R}} h_{R, E} v_{R} \\
& +\alpha \sqrt{P_{R}} \sqrt{P_{J}} h_{J_{1}, R} h_{R, E} j_{1}+\sqrt{P_{J}} h_{J_{2}, E} j_{2}+w_{E},
\end{aligned}
$$

where $w_{1}, w_{2}$, and $w_{E}$ represent the noise terms at $S_{1}, S_{2}$, and $E$, respectively. Then, $\Gamma_{j}$, defined as the overall signal to interference-plus-noise ratio (SINR) of the channel $S_{i} \rightarrow S_{j}$ (for $i, j=1,2, i \neq j$ ), can be calculated as

$$
\Gamma_{j}=\frac{\gamma_{S_{i}, S_{j}}}{\gamma_{J_{1}, S_{j}}+\gamma_{J_{2}, S_{j}}+\gamma_{R, S_{j}}+1},
$$

where $\gamma_{m, n}$ represents the instantaneous signal-to-noise ratio (SNR) for the link $m \rightarrow n$ :

$$
\begin{aligned}
& \gamma_{S_{i}, S_{j}}=\alpha^{2} P_{R} P_{S}\left|h_{S_{i}, R}\right|^{2}\left|h_{R, S_{j}}\right|^{2}, \\
& \gamma_{J_{1}, S_{j}}=\alpha^{2} P_{R} P_{J}\left|h_{J_{1}, R}\right|^{2}\left|h_{R, S_{j}}\right|^{2}, \\
& \gamma_{J_{2}, S_{j}}=P_{J}\left|h_{J_{2}, S_{j}}\right|^{2}, \\
& \gamma_{R, S_{j}}=\alpha^{2} P_{R}\left|h_{R, S_{j}}\right|^{2} .
\end{aligned}
$$


Strictly speaking, in order to maximize the overall SINR of the eavesdropping links, the eavesdropper $(E)$ can perform whatever operations as it wishes with the signals received in the previous two phases. Here in this paper, we take a simple case in which the eavesdropper applies maximal ratio combining (MRC) [34], so as to examine the efficiency of the proposed jamming schemes 1 . According to MRC, $E$ combines the received signals by multiplying $e_{1}$ in (2) and $e_{2}$ in (6) with proper weighting factors $a_{1}$ and $a_{2}$, respectively. Without loss of generality, consider the scenario in which $E$ intends to optimize the SINR of eavesdropper link $S_{i} \rightarrow E$, for $i=1,2$, the combined eavesdropping signal can be written as

$$
e^{i}=a_{1}^{i} e_{1}+a_{2}^{i} e_{2}
$$

where

$$
\begin{aligned}
& a_{1}^{i} \triangleq \frac{\sqrt{P_{S}} h_{S_{i}, E}^{H}}{\sigma_{N_{e_{1}}, S_{j}}^{2}}, \\
& a_{2}^{i} \triangleq \frac{\alpha \sqrt{P_{S}} h_{S_{i}, R}^{H} h_{R, E}^{H}}{\sigma_{N_{e_{2}}, S_{j}}^{2}},
\end{aligned}
$$

with $i, j=1,2, i \neq j$, and $(\cdot)^{H}$ is the conjugate transpose. $\sigma_{N_{e_{1}}, S_{j}}^{2}$ and $\sigma_{N_{e_{2}}, S_{j}}^{2}$ represent the total interference and noise power terms in $e_{1}$ and $e_{2}$, respectively:

$$
\begin{aligned}
& \sigma_{N_{e_{1}, S_{j}}}^{2}=\gamma_{S_{j}, E}+\gamma_{J_{1}, E}+1, \\
& \sigma_{N_{e_{2}, S_{j}}}^{2}=\gamma_{S_{j}, R, E}+\gamma_{J_{1}, R, E}+\gamma_{J_{2}, E}+\gamma_{R, E}+1,
\end{aligned}
$$

where

$$
\begin{aligned}
& \gamma_{S_{j}, E}=P_{S}\left|h_{S_{j}, E}\right|^{2}, \\
& \gamma_{J_{j}, E}=P_{J}\left|h_{J_{j}, E}\right|^{2}, \\
& \gamma_{S_{j}, R, E}=\alpha^{2} P_{R} P_{S}\left|h_{S_{j}, R}\right|^{2}\left|h_{R, E}\right|^{2}, \\
& \gamma_{J_{1}, R, E}=\alpha^{2} P_{R} P_{J}\left|h_{J_{1}, R}\right|^{2}\left|h_{R, E}\right|^{2}, \\
& \gamma_{R, E}=\alpha^{2} P_{R}\left|h_{R, E}\right|^{2} .
\end{aligned}
$$

In order to calculate the SINR of link $S_{i} \rightarrow E$, we assume two different channel knowledge sets:

${ }^{1}$ Please note that the eavesdropper's operation is not limited to maximal ratio combining (MRC). And the increasing in secrecy rates of the proposed schemes can still be achieved if the eavesdropper takes other operations, since the basic forms of the SINRs and thus of the secrecy rates do not change. 
1) $\psi_{0}$ that denotes a global instantaneous knowledge for all the links,

2) $\psi_{1}$ that denotes an average channel knowledge for the eavesdropper links.

With the assumption of $\psi_{0}$, we can get the instantaneous SNR of any channel $i \rightarrow j$ in the system. Thus, the SINR of link $S_{i} \rightarrow E$ can be calculated as

$$
\begin{aligned}
\Gamma_{E_{i}} & =\frac{P_{S}\left|h_{S_{i}, E}\right|^{2}}{\sigma_{N_{e_{1}}, S_{j}}^{2}}+\frac{\alpha^{2} P_{R} P_{S}\left|h_{S_{i}, R}\right|^{2}\left|h_{R, E}\right|^{2}}{\sigma_{N_{e_{2}}, S_{j}}^{2}} \\
& =\frac{\gamma_{S_{i}, E}}{\gamma_{S_{j}, E}+\gamma_{J_{1}, E}+1}+\frac{\gamma_{S_{i}, R, E}}{\gamma_{S_{j}, R, E}+\gamma_{J_{1}, R, E}+\gamma_{J_{2}, E}+\gamma_{R, E}+1},
\end{aligned}
$$

s.t. $\psi_{0}$.

In an environment where the instantaneous channel knowledge set $\psi_{0}$ is not available, we can use the expectation of SNRs for the eavesdropper links $\mathbb{E}\left[\gamma_{S_{i}, E}\right]$, which is provided by the average channel knowledge $\psi_{1}$, to get the SINRs:

$$
\Gamma_{E_{i}}^{\prime}=\frac{\mathbb{E}\left[\gamma_{S_{i}, E}\right]}{\mathbb{E}\left[\gamma_{S_{j}, E}\right]+\mathbb{E}\left[\gamma_{J_{1}, E}\right]+1}+\frac{\mathbb{E}\left[\gamma_{S_{j}, R, E}\right]}{\mathbb{E}\left[\gamma_{S_{j}, R, E}\right]+\mathbb{E}\left[\gamma_{J_{1}, R, E}\right]+\mathbb{E}\left[\gamma_{J_{2}, E}\right]+\mathbb{E}\left[\gamma_{R, E}\right]+1},
$$

s.t. $\psi_{1}$,

where $\mathbb{E}[\cdot]$ stands for the expectation operator.

\section{B. Problem Formulation}

The instantaneous secrecy rate for the node set $S_{i n}$ for source $S_{i}$ can be expressed [35]

$$
R_{S_{i}}\left(R, J_{1}, J_{2}\right)=\left[\frac{1}{2} \log _{2}\left(1+\Gamma_{i}\right)-\frac{1}{2} \log _{2}\left(1+\Gamma_{E_{j}}\right)\right]^{+},
$$

where $i=1,2, j=1,2, i \neq j$, and $[x]^{+} \triangleq \max \{0, x\}$.

The overall secrecy performance of the system is characterized by the ergodic secrecy capacity that is the expectation of the sum of the two sources' secrecy rates, $\mathbb{E}\left[R_{S}\left(R, J_{1}, J_{2}\right)\right]$, where

$$
R_{S}\left(R, J_{1}, J_{2}\right)=R_{S_{1}}\left(R, J_{1}, J_{2}\right)+R_{S_{2}}\left(R, J_{1}, J_{2}\right) .
$$

Our objective is to select appropriate nodes $R, J_{1}$, and $J_{2}$ in order to maximize the instantaneous secrecy rate subject to different types of channel feedback. The optimization problem can be formulated as

$$
\left(R^{*}, J_{1}^{*}, J_{2}^{*}\right)=\underset{\substack{R, J_{1}, J_{2} \in S_{i n} \\ R \neq J_{1}, J_{2}}}{\arg \max } R_{S}\left(R, J_{1}, J_{2}\right),
$$


where $u=0,1 ; R^{*}, J_{1}^{*}$ and $J_{2}^{*}$ denote the selected relay and jamming nodes, respectively. Note that here the selected jammers $J_{1}^{*}$ and $J_{2}^{*}$ in the two phases may be the same node, which is determined by the instantaneous secrecy rate.

\section{Selection without Jamming}

In a conventional cooperative network, the relay scheme does not have the help from jamming nodes. We derive the following solutions under this scenario.

1) Conventional Selection (CS): The conventional selection does not take the eavesdropper channels into account, and the relay node is selected according to the instantaneous SNR of the links between node $S_{1}$ and node $S_{2}$ only. Therefore, the SINR given in (7) becomes

$$
\Gamma_{j}^{C S}=\frac{\gamma_{S_{i}, S_{j}}}{\gamma_{R, S_{j}}+1}
$$

where $\Gamma_{j}^{C S}$ represents the SINR of the channel $S_{i} \rightarrow S_{j}$ (for $i, j=1,2, i \neq j$ ) without considering the eavesdropper.

Hence, the conventional selection algorithm can be expressed as

$$
\begin{aligned}
R^{*} & =\arg \max _{R \in S_{i n}}\left\{R_{S_{1}}(R)+R_{S_{2}}(R)\right\} \\
& =\arg \max _{R \in S_{i n}}\left\{\frac{1}{2} \log _{2}\left(1+\Gamma_{1}^{C S}\right)+\frac{1}{2} \log _{2}\left(1+\Gamma_{2}^{C S}\right)\right\} \\
& =\arg \max _{R \in S_{i n}}\left\{\left(1+\frac{\gamma_{S_{1}, S_{2}}}{\gamma_{R, S_{2}}+1}\right) \cdot\left(1+\frac{\gamma_{S_{2}, S_{1}}}{\gamma_{R, S_{1}}+1}\right)\right\},
\end{aligned}
$$

with $\gamma_{S_{i}, S_{j}}$ and $\gamma_{R, S_{j}}$ for $(i, j=0,1)$ given by (8) and (11), respectively. Since (28) shows that this selection does not consider the eavesdropping links, the CS algorithm may not able to support systems with the secrecy constraints even though it may effective in non-eavesdropper environments.

2) Optimal Selection (OS): This solution takes the eavesdropper into account and selects the relay node based on $\psi_{0}$, which provides the instantaneous channel knowledge for all the links. Then, the SINR of link $S_{i} \rightarrow E$ in (22) can be rewritten as

$$
\Gamma_{E_{i}}^{O S}=\frac{\gamma_{S_{i}, E}}{\gamma_{S_{j}, E}+1}+\frac{\gamma_{S_{i}, R, E}}{\gamma_{S_{j}, R, E}+\gamma_{R, E}+1} .
$$

The optimal selection is given as:

$$
\begin{aligned}
R^{*} & =\arg \max _{R \in S_{i n}}\left\{R_{S_{1}}(R)+R_{S_{2}}(R)\right\} \\
& =\arg \max _{R \in S_{i n}}\left\{\frac{1}{2} \log _{2}\left(1+\Gamma_{1}^{O S}\right)-\frac{1}{2} \log _{2}\left(1+\Gamma_{E_{2}}^{O S}\right)+\frac{1}{2} \log _{2}\left(1+\Gamma_{2}^{O S}\right)-\frac{1}{2} \log _{2}\left(1+\Gamma_{E_{1}}^{O S}\right)\right\} \\
& =\arg \max _{R \in S_{i n}}\left\{\frac{1+\Gamma_{1}^{O S}}{1+\Gamma_{E_{2}}^{O S}} \cdot \frac{1+\Gamma_{2}^{O S}}{1+\Gamma_{E_{1}}^{O S}}\right\},
\end{aligned}
$$


where

$$
\Gamma_{i}^{O S}=\Gamma_{i}^{C S}=\frac{\gamma_{S_{j}, S_{i}}}{\gamma_{R, S_{i}}+1} .
$$

3) Suboptimal Selection (SS): The suboptimal selection implements the relay selection based on the knowledge set $\psi_{1}$, which gives the average estimate of the eavesdropper links. Therefore, it avoids the difficulty of getting instantaneous estimate of the channel feedbacks. Similar to the OS algorithm in (30), the suboptimal selection can be written as

$$
R^{*}=\arg \max _{R \in S_{i n}}\left\{\frac{1+\Gamma_{1}^{S S}}{1+\Gamma_{E_{2}}^{S S}} \cdot \frac{1+\Gamma_{2}^{S S}}{1+\Gamma_{E_{1}}^{S S}}\right\},
$$

where

$$
\begin{aligned}
\Gamma_{i}^{S S} & =\Gamma_{i}^{O S}=\frac{\gamma_{S_{j}, S_{i}}}{\gamma_{R, S_{i}}+1}, \\
\Gamma_{E_{i}}^{S S} & =\frac{\mathbb{E}\left[\gamma_{S_{i}, E}\right]}{\mathbb{E}\left[\gamma_{S_{j}, E}\right]+1}+\frac{\mathbb{E}\left[\gamma_{S_{i}, R, E}\right]}{\mathbb{E}\left[\gamma_{S_{j}, R, E}\right]+\mathbb{E}\left[\gamma_{R, E}\right]+1} .
\end{aligned}
$$

Note that in comparison of the OS in (30), the only difference of the SS algorithm in (32) is that it requires the average channel state information, $\psi_{1}$, which would be more useful in practice.

\section{Selections With JAMming In TwO-WAY Relay Systems}

In this section, we present several node selection techniques based on the optimization problem given by (26) in the two-way systems. Unlike [31], where the selection techniques only concern about the secrecy performance in the second phase of transmission, here, our work takes into account both the two phases in order to select a set of relay and jammers that can maximize the overall expectation of secrecy rate.

\section{A. Optimal Selection with Maximum Sum Instantaneous Secrecy Rate (OS-MSISR)}

The optimal selection with maximum sum instantaneous secrecy rate assumes a knowledge set $\psi_{0}$ and ensures a maximization of the sum of instantaneous secrecy rates of node $S_{1}$ and node $S_{2}$ given in (25), which gives credit to

$$
\begin{aligned}
\left(R^{*}, J_{1}{ }^{*}, J_{2}{ }^{*}\right) & =\underset{\substack{R, J_{1} J_{2} \in S_{i n} \\
R \neq J_{1}, J_{2}}}{\arg \max }\left\{R_{S}\left(R, J_{1}, J_{2}\right)\right\} \\
& =\underset{\substack{\text { arg } \max \\
R, J_{1}, J_{2} \in S_{i n} \\
R \neq J_{1}, J_{2}}}{\arg \operatorname{man}}\left\{\frac{1+\Gamma_{2}}{1+\Gamma_{E_{1}}} \cdot \frac{1+\Gamma_{1}}{1+\Gamma_{E_{2}}}\right\},
\end{aligned}
$$

where $\Gamma_{i}$ and $\Gamma_{E_{i}}$ are given by (7) and (22), respectively. 
The approach in (35) reflects the basic idea of using both cooperative relaying and cooperative jamming in order to promote the system's secrecy performance. Specifically, the OS-MSISR scheme here tends to select a set of relay and jammers that maximizes $\Gamma_{i}$, which means promoting the assistance to the sources. Meanwhile this relay and jammer set tends to minimize $\Gamma_{E_{i}}$, which is equivalent to enhance the interference to the eavesdropper.

Although the OS-MSISR scheme seems to be a straightforward application for cooperative relaying and cooperative jamming, the actual selection procedure usually involves tradeoffs. For instance, according to (7) and (9), we should select the relay and jammer set that minimizes $\left|h_{J_{1}, R}\right|$ in order to make $\Gamma_{i}$ as high as possible. Considering (19), (20) and (22), however, the lower $\left|h_{J_{1}, R}\right|$ is, the higher $\Gamma_{E_{i}}$ is, which is undesirable. Thus, we have to make a trade-off between raising $\Gamma_{i}$ and inhibiting $\Gamma_{E_{i}}$ in order to optimize the right part of (35).

\section{B. Optimal Selection with Max-Min Instantaneous Secrecy Rate (OS-MMISR)}

It is obvious that the OS-MSISR in (35) is complicated, in this subsection we propose a reduced-complexity algorithm. It is common that the sum secrecy rate of two sources, i.e. $R_{S_{1}}\left(R, J_{1}, J_{2}\right)+R_{S_{2}}\left(R, J_{1}, J_{2}\right)$, may be driven down to a low level by the user with the lower secrecy rate. As a result, for low complexity, the intermediate nodes, which maximize the minimum secrecy rate of two users, can be selected to achieve the near-optimal performance. In addition, in some scenarios, the considered secrecy performance does not only take into account the total secrecy rate of all the source nodes, but also the individual secrecy rate of each node. If one source node has low secrecy rate, the whole system is regarded as secrecy inefficient. Furthermore, assuring each individual source node a high secrecy rate is another perspective of increasing the whole system's secrecy performance.

The OS-MMISR selection maximizes the worse instantaneous secrecy rate of the two source nodes with the assumption of knowledge set $\psi_{0}$, and we can get

$$
\begin{aligned}
\left(R^{*}, J_{1}{ }^{*}, J_{2}{ }^{*}\right) & =\underset{\substack{R, J_{1}, J_{2} \in S_{i n} \\
R \neq J_{1}, J_{2}}}{\arg \max \min }\left\{R_{S_{1}}\left(R, J_{1}, J_{2}\right), R_{S_{2}}\left(R, J_{1}, J_{2}\right)\right\} \\
& =\underset{\substack{R, J_{1}, J_{2} \in S_{i n} \\
R \neq J_{1}, J_{2}}}{\arg \max \min }\left\{\frac{1+\Gamma_{2}}{1+\Gamma_{E_{1}}}, \frac{1+\Gamma_{1}}{1+\Gamma_{E_{2}}}\right\}
\end{aligned}
$$

where $\Gamma_{i}$ and $\Gamma_{E_{i}}$ are given by (7) and (22), respectively. 


\section{Optimal Switching (OSW)}

The original idea of using jamming nodes is to introduce interference on the eavesdropper links. However, there are two side-effects of using jamming. Firstly, the jamming node in the second phase $J_{2}$ poses undesired interference directly onto the destinations. Secondly, it degrades the links between the relay node $R$ and the destinations. Given the assumption that the destinations cannot mitigate this artificial interference, continuous jamming in both phases is not always beneficial for the whole system. In some specific situation (e.g., $J_{2}$ is close to one destination), the continuous jamming may decrease secrecy rate seriously, and act as a bottleneck for the system. In order to overcome this problem, we introduce the idea of intelligent switching between the OS-MSISR and the OS scheme in order to reduce the impact of "negative interference". The threshold for the involvement of the jammer nodes is

$$
R_{S_{1}}\left(R, J_{1}, J_{2}\right)+R_{S_{2}}\left(R, J_{1}, J_{2}\right)>R_{S_{1}}^{O S}(R)+R_{S_{2}}^{O S}(R),
$$

where

$$
R_{S_{i}}^{O S}(R)=\left[\frac{1}{2} \log _{2}\left(\frac{1+\Gamma_{i}^{O S}}{1+\Gamma_{E_{j}}^{O S}}\right)\right]^{+} .
$$

Thus, (37) can be further written as

$$
\frac{1+\Gamma_{1}}{1+\Gamma_{E_{2}}} \cdot \frac{1+\Gamma_{2}}{1+\Gamma_{E_{1}}}>\frac{1+\Gamma_{1}^{O S}}{1+\Gamma_{E_{2}}^{O S}} \cdot \frac{1+\Gamma_{2}^{O S}}{1+\Gamma_{E_{1}}^{O S}},
$$

where $\Gamma_{i}, \Gamma_{E_{i}}, \Gamma_{i}^{O S}$ and $\Gamma_{E_{i}}^{O S}$ are given by (7) and (22), (31) and (29), respectively.

For each time slot, if (39) is met, the OS-MSISR scheme provides higher instantaneous secrecy rate than OS does and is preferred. Otherwise the OS scheme is more efficient in promoting the system's secrecy performance, which should be employed. Because of the uncertainty of the channel coefficient $h_{i, j}$ for each channel $i \rightarrow j$, the OSW should outperform either the continuous jamming scheme or the non-jamming one.

\section{Suboptimal Selection with Maximum Sum Instantaneous Secrecy Rate (SS-MSISR)}

With the assumption of $\psi_{0}$, we can get some optimal selection metrics. However, its practical interest and potential implements are only limited to some special (e.g. military) applications, where the instantaneous quality of the eavesdropper links can be measured by some specific protocols. In practice, only an average knowledge of these links $\psi_{1}$ would be available from long term eavesdropper supervision. The selection metrics is modified as 


$$
\left(R^{*}, J_{1}{ }^{*}, J_{2}{ }^{*}\right)=\underset{\substack{R, J_{1}, J_{2} \in S_{i n} \\ R \neq J_{1}, J_{2}}}{\arg \max }\left\{\frac{1+\Gamma_{2}}{1+\Gamma_{E_{1}}^{\prime}} \cdot \frac{1+\Gamma_{1}}{1+\Gamma_{E_{2}}^{\prime}}\right\},
$$

where $\Gamma_{i}$ and $\Gamma_{E_{i}}^{\prime}$ are given by (7) and (23), respectively.

From (40), we can predict that for a scenario in which the intermediate nodes are sparsely distributed across the considered area, the SS-MSISR scheme can provide similar relay and jammer selection with the OS-MSISR scheme. This is because a slightly difference between $\mathbb{E}\left[\gamma_{i, E}\right]$ provided by $\psi_{1}$ and $\gamma_{i, E}$ provided by $\psi_{0}$ would not be enough for the scheme to select another far-away intermediate node. Thus, under this condition, the average eavesdropper channel knowledge set $\psi_{1}$ may contain sufficient channel information as well for a quasioptimal selection.

\section{E. Suboptimal Selection with Max-Min Instantaneous Secrecy Rate (SS-MMISR)}

This scheme refers to the practical application of the above selection with maximum worse instantaneous secrecy rate in (36). The basic idea of considering $\psi_{1}$ as the average behavior of eavesdropper links is the same as SS-MSISR, but aimed at looking for the maximum worse instantaneous secrecy rate, which is written as

$$
\begin{aligned}
\left(R^{*}, J_{1}{ }^{*}, J_{2}{ }^{*}\right) & =\underset{\substack{R, J_{1}, J_{2} \in S_{i n} \\
R \neq J_{1}, J_{2}}}{\arg \max \min }\left\{R_{S_{1}}\left(R, J_{1}, J_{2}\right), R_{S_{2}}\left(R, J_{1}, J_{2}\right)\right\} \\
& =\underset{\substack{R, J_{1}, J_{2} \in S_{i n} \\
R \neq J_{1}, J_{2}}}{\arg \max \min }\left\{\frac{1+\Gamma_{2}}{1+\Gamma_{E_{1}}^{\prime}}, \frac{1+\Gamma_{1}}{1+\Gamma_{E_{2}}^{\prime}}\right\},
\end{aligned}
$$

where $\Gamma_{i}$ and $\Gamma_{E_{i}}^{\prime}$ are given by (7) and (23), respectively.

\section{F. Suboptimal Switching (SSW)}

Given the fact that jamming is not always a positive process for the performance of the system, the suboptimal switching refers to the practical application of the intelligent switching between the SS-MSISR and the SS schemes. The basic idea is the same as OSW, but the switching criterion uses the available knowledge set $\psi_{1}$. More specifically, the required condition for switching from SS-MSISR to SS mode is

$$
\frac{1+\Gamma_{1}}{1+\Gamma_{E_{2}}^{\prime}} \cdot \frac{1+\Gamma_{2}}{1+\Gamma_{E_{1}}^{\prime}}>\frac{1+\Gamma_{1}^{S S}}{1+\Gamma_{E_{2}}^{S S}} \cdot \frac{1+\Gamma_{2}^{S S}}{1+\Gamma_{E_{1}}^{S S}},
$$

where $\Gamma_{i}, \Gamma_{E_{i}}^{\prime}, \Gamma_{i}^{S S}$ and $\Gamma_{E_{i}}^{S S}$ are given by (7) and (23), (33) and (34), respectively. 


\section{G. Optimal Selection with “Known” Jamming (OSKJ)}

The previous selection techniques are proposed based on the assumption that the jamming signal is unknown at both the two destinations. This assumption avoids the initialization period in which the jamming sequence is defined, and thus, it reduces the risk of giving out the artificial interference to the eavesdropper. For comparison reasons, here we propose a "control" scheme, in which the jamming signal can be decoded at the destinations $S_{1}$ and $S_{2}$, but not at the eavesdropper $E$. In this case, the SINR of the link from $S_{i}$ (for $i=1,2$ ) to $E$ remains the same as $\Gamma_{E_{i}}$ given by (22). The SINR of the link from $S_{i}$ to $S_{j}$ (for $i, j=1,2, i \neq j$ ) is modified as follows:

$$
\Gamma_{i}=\frac{\gamma_{S j, S i}}{\gamma_{R, S i}+1}
$$

The OSKJ scheme is taken into consideration in the numerical results section as a reference. This, however, is not the "ideal" jamming scheme since the artificial interference from the jammers only degrades the eavesdropper links. As we have discovered and will discuss in Section $\mathrm{V}$, in some particular scenarios, the OSKJ scheme is outperformed by the OSW and SSW schemes presented above, for the jamming has changed the value of $\alpha$ given in (3).

\section{Performance Analysis}

In this section, we firstly do some quantitative analysis on the asymptotic performance of both the proposed jamming and non-jamming schemes in high transmitted power range. Then, we provide a qualitative discussion of the secrecy performance of different selection schemes in some typical scenarios based on the system model in Section II.

\section{A. Asymptotic Performance for Selections without Jamming}

Without loss of generality, we take the OS scheme for example. With high transmitted power $P_{S}$, we can get

$$
\begin{aligned}
& \Gamma_{i}^{O S} \rightarrow P_{S}\left|h_{S_{i}, R}\right|^{2}, \\
& \Gamma_{E_{i}}^{O S} \rightarrow \frac{\left|h_{S_{i}, E}\right|^{2}}{\left|h_{S_{j}, E}\right|^{2}}+\frac{\left|h_{S_{i}, R}\right|^{2}}{\left|h_{S_{j}, R}\right|^{2}} .
\end{aligned}
$$

We can see that $\Gamma_{i}^{O S}$ grows rapidly as $P_{S}$ increases, while $\Gamma_{E_{i}}^{O S}$ converges to a value that depends only on the relative distances between the sources, the eavesdropper and the relay. Therefore, the ergodic secrecy capacity $\mathbb{E}\left[R_{S}\right]$ also increases rapidly with the transmitted 
power $P_{S}$. Based on (44) and (45), the slope of the curve of $\mathbb{E}\left[R_{S}\right]$ versus $P_{S}$ (measured by dB) can be approximately calculated as

$$
\begin{aligned}
& \frac{\partial \mathbb{E}\left[R_{S_{1}}+R_{S_{2}}\right]}{\partial P_{S}} \\
= & \frac{\partial \mathbb{E}\left[\frac{1}{2} \log _{2} \frac{10^{P_{S} / 10}\left|h_{S 1, R}\right|^{2} \cdot 10^{P_{S}} / 10\left|h_{S 2, R}\right|^{2}}{\Gamma_{E 1}^{O S} \cdot \Gamma_{E 2}^{O S}}\right]}{\partial P_{S}} \\
= & \frac{\partial \mathbb{E}\left[\frac{1}{2} \log _{2} 10^{2 P_{S} / 10}\right]}{\partial P_{S}}+\frac{\partial \mathbb{E}\left[\frac{1}{2} \log _{2} \frac{\left|h_{S 1, R}\right|^{2}\left|h_{S 2, R}\right|^{2}}{\Gamma_{E 1}^{O S} \cdot \Gamma_{E 2}^{O S}}\right]}{\partial P_{S}} \\
= & \frac{\partial\left(\frac{P_{S}}{10} \log _{2} 10\right)}{\partial P_{S}} \\
= & \frac{1}{10} \log _{2} 10 \\
\approx & 0.3322
\end{aligned}
$$

For the other non-jamming schemes (i.e. CS, SS), we note that they share the same asymptotic performance as the OS scheme with a linear increment of slope about 0.3322 as the transmitted power $P_{S}$ increases.

\section{B. Asymptotic Analysis for Selections with Continuous Jamming}

We use the same method as in the previous analysis for the non-jamming selections to analyze the asymptotic performance of the proposed jamming schemes. As the transmitted power $P_{S}$ increases to a relatively high value, it yields

$$
\begin{gathered}
\lim _{P_{S} \rightarrow \infty} \Gamma_{i}=\frac{L\left|h_{S_{j}, R}\right|^{2}\left|h_{R, S_{i}}\right|^{2}}{\left|h_{J_{1}, R}\right|^{2}\left|h_{R, S_{i}}\right|^{2}+\left|h_{S_{1}, R}\right|^{2}\left|h_{J_{2}, S_{i}}\right|^{2}+\left|h_{S_{2}, R}\right|^{2}\left|h_{J_{2}, S_{i}}\right|^{2}}, \\
\lim _{P_{S} \rightarrow \infty} \Gamma_{E_{i}}=\frac{\left|h_{S_{i}, E}\right|^{2}}{\left|h_{S_{j}, E}\right|^{2}}+\frac{\left|h_{S_{i}, R}\right|^{2}}{\left|h_{S_{j}, R}\right|^{2}} .
\end{gathered}
$$

It is clear that both $\Gamma_{i}$ and $\Gamma_{E_{i}}$ are independent of $P_{S}$, which means that for high $P_{S}$, the ergodic secrecy rate $\mathbb{E}\left[R_{S}\right]$ stops increasing and converges to a fixed value. Consider the asymptotic performance of the OS scheme that grows linearly with the increment of $P_{S}$ as described by (46), it is safe to predict that there will be a crossover point, $P^{\prime}$, between the ergodic secrecy rate v.s. transmitted power curve with jamming and the one with nonjamming. In a power range below $P^{\prime}$, the jamming scheme outperforms the non-jamming one, while above this point, the jamming scheme loses its advantage in providing higher ergodic secrecy capacity. 
We note that the analysis above can apply to any scheme with continuous jamming (i.e., OSMSISR, OS-MMISR, SS-MSISR, and SS-MMISR), which indicates that they share the same asymptotic behavior as the $P_{S}$ increases. In another word, the proposed selection techniques (except for OSW and SSW) behave better than the non-jamming schemes only within a certain transmitted power range. Fortunately, in a practical case, $P_{S}$ is always limited in a relatively low range and will not increase infinitely.

\section{Secrecy Performance with Sparsely Distributed Intermediate Nodes}

This is a common configuration in which the the eavesdropper $E$ has similar distance with two sources $S_{1}$ and $S_{2}$ and the intermediate nodes spread randomly within the considered area. With a relatively far distance in between, the interference link between $J_{1}$ and $R$ becomes weak. As predicted in the previous subsection, within a certain transmitted power range (less than the crossover point $P^{\prime}$ ), the selection approaches with continuous jamming are able to provide a higher ergodic secrecy rate than the non-jamming schemes. This gain proves the introduction of jamming in selection schemes as an effective technique. Outside this range, the secrecy rates of the conventional non-jamming schemes continue to grow with a slope of 0.3322 as verified by (46), whereas those of the continuous jamming schemes converge to a fixed value. Inside this scope, the jamming schemes lose their efficiency in providing a better secrecy performance for the system.

We note that in some particular scenarios, the system's integrated secrecy performance is not measured by the sum of the total secrecy rates, but by the minimum secrecy capacity of all the source nodes in the system. In this situation, OS-MMISR and SS-MMISR can optimize the overall secrecy performance of the whole system. For the hybrid schemes, the OSW and SSW schemes are able to provide better secrecy performance in the whole transmitted power scope, since it overcomes the bottleneck caused by negative interference on the relay-destination links.

\section{Secrecy Performance With a Close Cluster of the Intermediate Nodes}

Under the condition that all the intermediate nodes are located very close to each other, we note that the continuous jamming selections will lose its efficiency in meeting the secrecy constraints. Specifically, we will discuss two extreme situations in which the intermediate nodes cluster is near to one of the destination nodes $S_{i}$, and to the eavesdropper $E$, respectively. 
1) The intermediate nodes cluster locates near to one of the destinations: There are two reasons that make the proposed jamming schemes inefficient. Firstly, the nodes of the relay/jammer cluster gather too close to each other, such that the selected jammer in the first phase $J_{1}$ has too much negative impact on the selected relay $R$, which further decreases the SINRs in the second phase. Secondly, the jamming code from $J_{2}$ in the second phase also has an overly-strong interference on the destination to the one it stays close with.

2) The intermediate nodes cluster locates near to the eavesdropper: Aside from the first reason presented above, in this configuration, the direct link between the relay $R$ and the eavesdropper $E$ gets too strong, which will seriously sabotage the secrecy performance of selection with continuous jamming.

On the other hand, the hybrid protocols (OSW and SSW) will still be the most effective schemes in this configuration, since the system's secrecy performance considered here is measured by the ergodic secrecy rate.

\section{E. Secrecy Performance With the Eavesdropper Near to One of the Source Nodes}

This is the situation in which the eavesdropper $E$ can get the communicating information most easily, since the direct link between $E$ and any one of the source nodes is strong, which makes the introduction of jamming very necessary. The jamming schemes should be efficient within quite a large power range, and the hybrid schemes should still perform as the best selection techniques within the whole power scope.

\section{NUMERICAL RESULTS}

In this section, we will provide computer simulations in order to validate the analysis in the previous section. The simulation environment takes into account two sources $S_{1}$ and $S_{2}$, one eavesdropper $E$, and a intermediate node cluster consisting of $K=8$ nodes. All the nodes are located in a $2 \mathrm{D}$ square topology within a $1 \times 1$ unit square. For simplicity, the source nodes and the relay transmit with the same power, i.e. $P_{S}=P_{R}$. The relay and jammer nodes transmit with a relay-jammer power ratio $L=10$. As assumed in Section II, the power of the AWGN is $\sigma^{2}=1$. The path-loss exponent is set to $\beta=3$. In this paper, the adopted performance metric is the ergodic secrecy rate. Meanwhile some results are also provided in terms of secrecy outage probability $\mathbb{P}\left[R_{S}\left(R^{*}, J_{1}^{*}, J_{2}^{*}\right)<R_{T}\right]$, where $\mathbb{P}[\cdot]$ denotes probability, and $R_{T}$ is the target secrecy rate. 


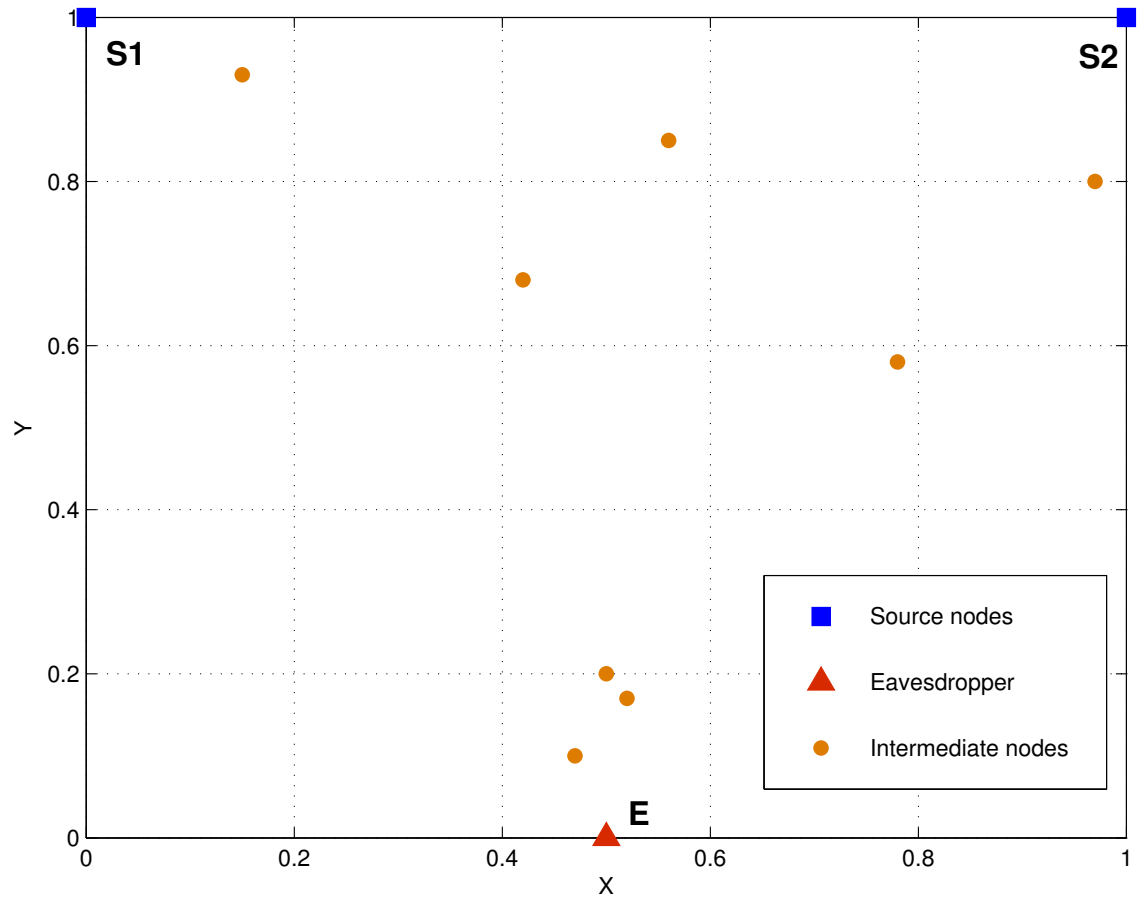

Fig. 2. The $1 \times 1$ simulation environment with $K=8, \beta=3$.

In the first simulation, we assume a scenario where $S_{1}, S_{2}$ and $E$ are located at $\left(X_{E}, Y_{E}\right)=$ $(0.5,0),\left(X_{S_{1}}, Y_{S_{1}}\right)=(0,1)$ and $\left(X_{S_{2}}, Y_{S_{2}}\right)=(1,1)$, respectively. The intermediate nodes spread randomly within the square space, as shown in Fig. 2.

Fig. 3 shows the ergodic secrecy rate versus the transmitted power $P_{S}=P_{R}$ of different selection schemes. We can observe that selection algorithms with jamming outperform their non-jamming counterparts within a certain transmitted power range (less than $P^{\prime} \approx 16 d B$ ), where the ergodic secrecy rate of the OS-MSISR scheme is approximately higher than that of the OS scheme by 1 bit per channel use (BPCU). Outside this range $\left(P>P^{\prime}\right)$, the secrecy rate of OS-MSISR converges to a power-independent value which is approximately 4.1 BPCU, whereas the ergodic secrecy rate of OS continues to grow with a slope of 0.3322 , as proved by (46). This validates the secrecy performance analysis in Section IV. In addition, we can see that in this relay topology, the suboptimal schemes (SS-MSISR, SS-MMISR) which are based on average channel knowledge perform almost the same as the optimal schemes (OSMSISR, OS-MMISR), which implies that in this configuration where the intermediate nodes are sparsely distributed, an average channel knowledge may also provide enough information in order to get optimal relay selection. 


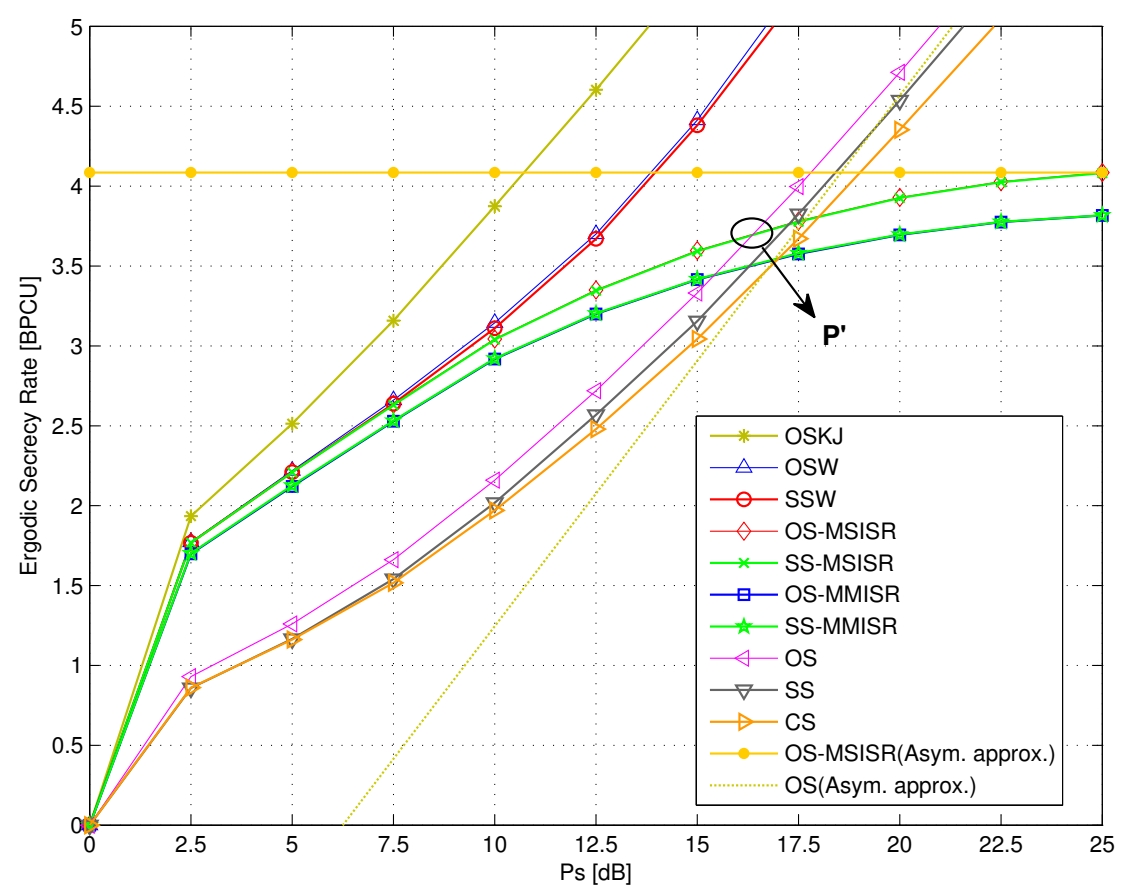

Fig. 3. Ergodic secrecy rate versus transmitted power $P_{S}$ for different selection techniques.

In Fig. 3, a comparison between the OS-MSISR and OS-MMISR shows that the OSMSISR scheme has slightly higher ergodic secrecy capacity by about 0.25 BPCU than OSMMISR does corresponding to transmitted power $P_{S}$. The same comparison result can be observed from the SS-MSISR and SS-MMISR schemes, which matches our previous analysis. Furthermore, it can be seen that OSW performs better than any other selection techniques with or without continuous jamming. At a low power range where $P_{S}<P^{\prime}$, the OSW scheme performs slightly better than OS-MSISR, but much better than OS (by about 1.2 BPCU), for the reason that in this range continuous jamming is almost always needed. After $P_{S}$ grows much higher than $P^{\prime}$, OSW outperforms both the other two schemes by a large scale. For the suboptimal case, we can see that SSW provides almost the same performance as the OSW scheme in this relay topology, which validates the practical value of this hybrid scheme. An observation of the performance of OSKJ scheme shows that it outperforms all the other selection techniques, providing the highest ergodic secrecy rate when the transmitted power increases due to its ability of the destinations to decode the artificial interference in this OSKJ scheme.

Within this configuration, we also compare the performance of different selection tech- 


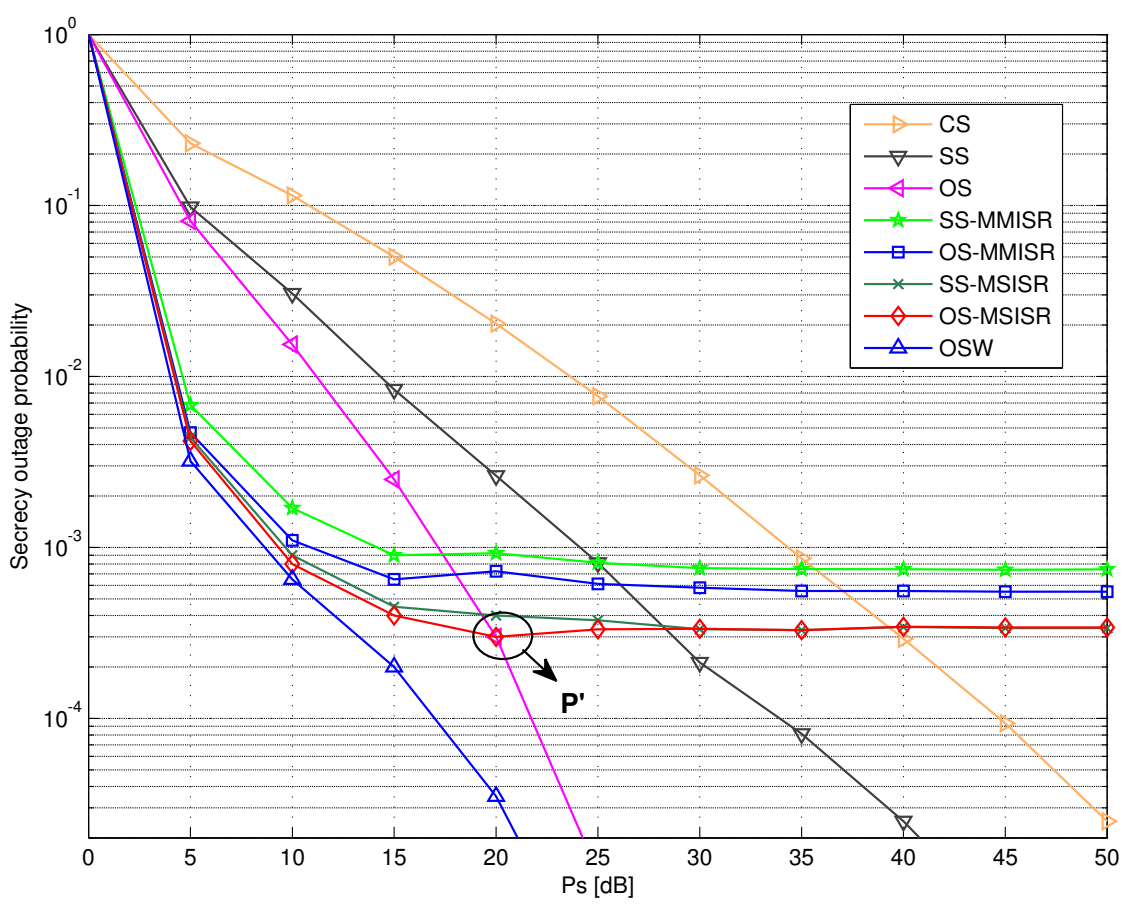

Fig. 4. Secrecy outage probability versus transmitted power $P_{S}$ for different selection techniques with $R_{T}=0.2$ BPCU.

niques measured by secrecy outage probability, which is shown in Fig. 4, The target secrecy rate $R_{S}$ is set as $0.2 \mathrm{BPCU}$. It can be seen that selection schemes with jamming provies lower secrecy outage probability within a certain transmitted power range $\left(P_{S}<P^{\prime}, P^{\prime} \approx 20 d B\right)$. Outside this range, the conventional selection without jamming achieves better secrecy outage probability. Regarding the hybrid protocols, the OSW scheme outperforms the non-switching selection techniques.

In Fig. 5, it deals with a configuration where the intermediate nodes cluster, which also includes $K=8$ nodes, is located closely near to one of the two users (e.g., node $S_{1}$, without loss of generality). We can see the ergodic secrecy rate of the proposed selection schemes in this topology differs greatly from that in the previous configuration. We observe that continuous jamming schemes (i.e. OS-MSISR, OS-MMISR, SS-MSISR, and SS-MMISR) are inefficient here, which converge to less than 0.5 BPCU, validating our discussion in Section IV.

On the other hand, OSW and SSW still outperform all the other selection techniques by a quite large scale (more than $4 \mathrm{BPCU}$ when $P_{S}$ is very high, as shown in Fig. 5, We also note that in this topology, the OSW and SSW schemes perform even better than the 


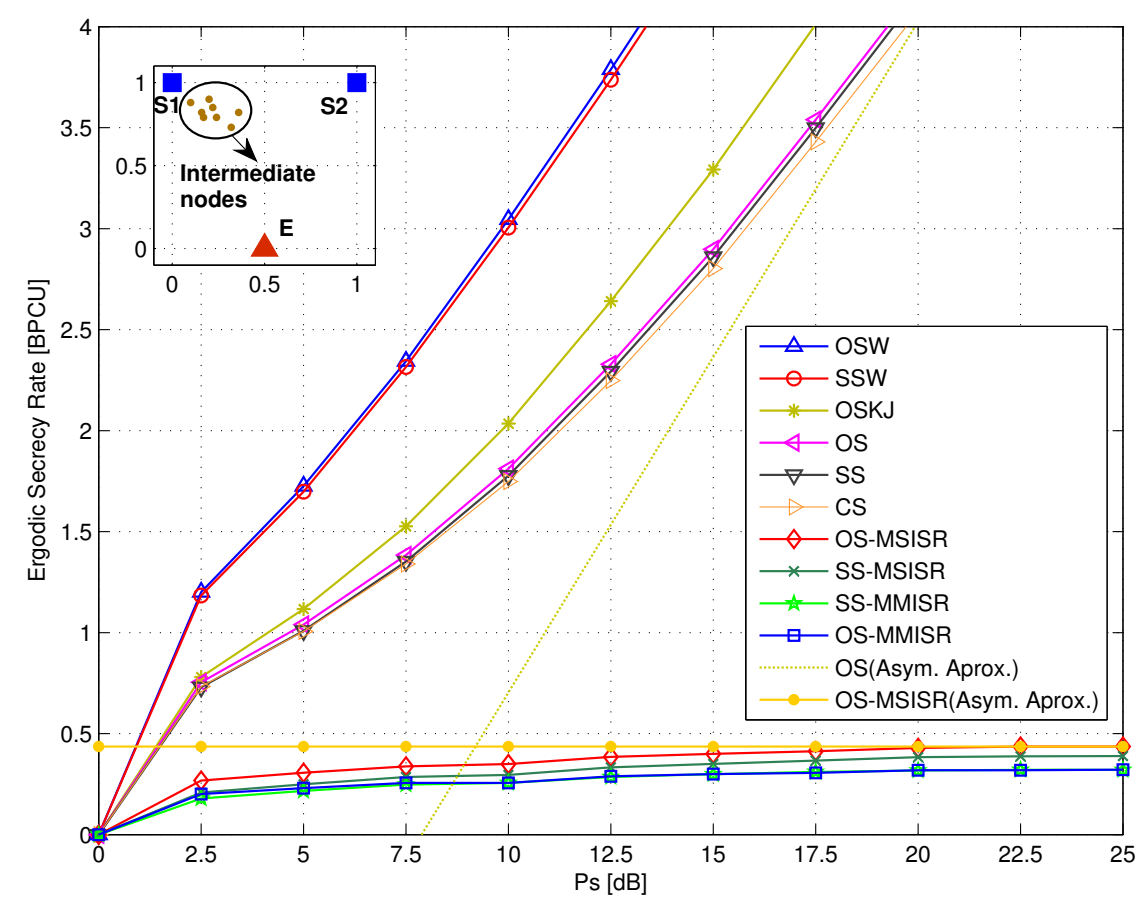

Fig. 5. Ergodic secrecy rate for a scenario where the intermediate nodes are close to $S_{1}$.

OSKJ scheme, which seems to be an interesting result. Further investigation reveals that the involvement of $J_{1}$ node in OSKJ causes a different value of $\alpha$ with that of OSW and SSW, which results in lower secrecy rates in OSKJ than in OSW and SSW schemes. This indicates that the proposed OSW/SSW schemes may perform even better than the "ideal" case where the destinations can mitigate the artificial interference. All of these validate the value of the selection techniques with intelligent switching in potential practical use.

In Fig. 6, we set the intermediate nodes cluster closely to the eavesdropper E. Here the jamming schemes also perform worse than non-jamming ones in most of the transmitted power range. It also shows the range where continuous jamming schemes perform better than non-jamming schemes in this topology is slightly larger than that of the previous one, since there is no strong $R \rightarrow E$ link here. Regarding to the hybrid schemes, OSW and SSW still perform as the best selection techniques in providing the highest secrecy rate.

Finally, we place the eavesdropper $E$ near to one of the two sources (taken $S_{1}$ for example) to examine the results. The location of eavesdropper $E$ is set to $\left(X_{E}, Y_{E}\right)=(0,0.5)$, the intermediate nodes are spread randomly across the considered rectangle area, as shown in the inset of Fig. 7 . We get a similar simulation result with that of the first configuration, 


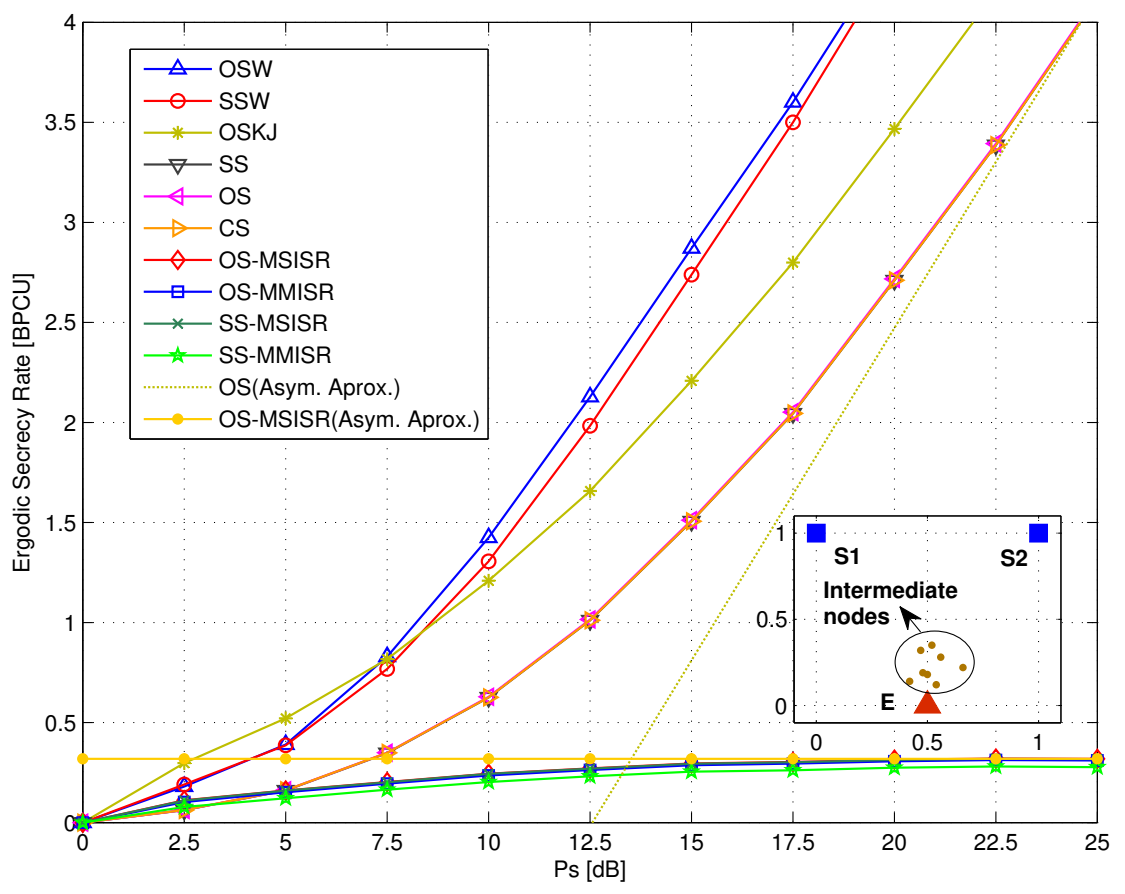

Fig. 6. Ergodic secrecy rate for a scenario where the intermediate nodes are close to the eavesdropper $E$.

in which the eavesdropper $E$ has the same distance with $S_{1}$ and $S_{2}$. The non-jamming schemes (CS, OS and SS) here are less effective in promoting the secrecy performance. On the contrary, the selection techniques with continuous jamming (OS-MSISR, OS-MMISR, SS-MSISR and SS-MMISR) provide much higher secrecy capacity in a large transmitted power range $\left(P^{\prime} \approx 13 d B\right)$. Within this power range, the hybrid schemes (OSW and SSW) perform slightly better than the continuous jamming techniques because jamming is almost always needed in this configuration. Outside this regime, where the non-jamming scheme performs better, the difference between the intelligent switching and continuous jamming increases and the hybrid schemes still perform as the most efficient schemes.

\section{CONCLUSIONS}

This paper has studied the joint relay and jammer selection in two-way cooperative networks with physical layer secrecy consideration. The proposed scheme achieves an opportunistic selection of one conventional relay node and one (or two) jamming nodes to increase security against eavesdroppers based on both instantaneous and average knowledge of the eavesdropper channels. The selected relay node helps enhance the information transmission 


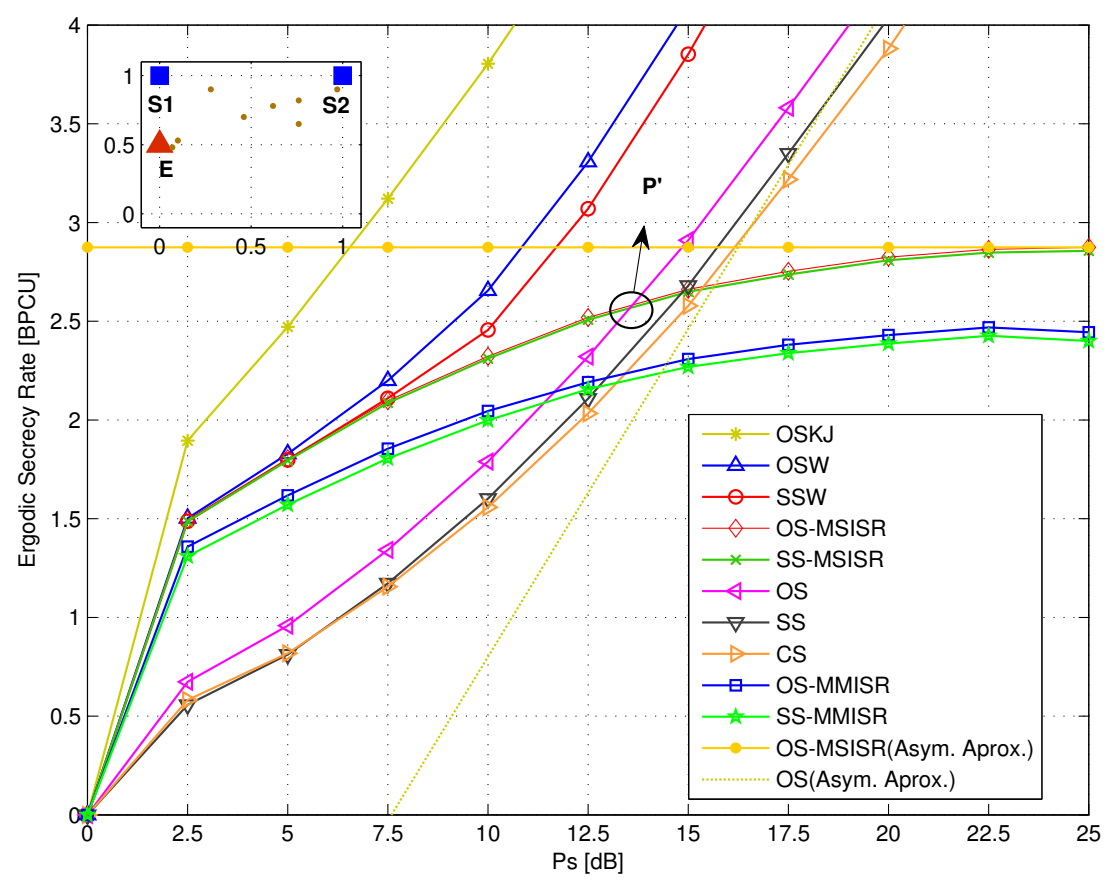

Fig. 7. Ergodic secrecy rate for a scenario where the eavesdropper $E$ is close to $S_{1}$.

between the two sources via an AF strategy, while the jamming nodes are used to produce intentional interference at the eavesdropper nodes in different transmission phases. We found that the proposed jamming schemes (i.e. OS-MSISR, OS-MMISR, SS-MSISR, and SSMMISR) are effective within a certain transmitted power range for scenarios with sparsely distributed intermediate nodes. Meanwhile the non-jamming schemes (CS, OS, and SS) are preferred in configurations where the intermediate nodes are confined close to each other. The OSW scheme which switches intelligently between jamming and non-jamming modes is very efficient in providing the highest secrecy rate in almost the whole transmitted power regime in two-way cooperative networks, but it requires an instantaneous eavesdropper channel knowledge. On the other hand, the suboptimal switching scheme, SSW, which is based on the average knowledge of the eavesdropper channel and therefore much practical, provides a comparable secrecy performance with the OSW scheme.

\section{REFERENCES}

[1] E. D. Silva, A. L. D. Santos, L. C. P. Albini, and M. Lima, "Identity-based key management in mobile ad hoc networks: techniques and applications," IEEE Wireless Communicaiton, vol. 15, no. 5, pp. 46-52, Oct. 2008.

[2] A. D. Wyner, “The wire-tap channel," Bell System Technical Journal, vol. 54, no. 8, pp. 1355-1387, Oct. 1975. 
[3] S. K. Leung-Yan-Cheong and M. E. Hellman, "The Gaussian wiretap channel," IEEE Transactions on Information Theory, vol. 24, no. 4, pp. 451-456, Jul. 1978.

[4] I. Csiszár and J. Körner, "Broadcast channels with confidential messages," IEEE Transactions on Information Theory, vol. 24, no. 3, pp. 339-348, May 1978.

[5] P. Parada and R. Blahut, "Secrecy capacity of SIMO and slow fading channels," in Proceedings of IEEE International Symposium on Information Theory, Adelaide, Australia, Sep. 2005.

[6] J. Barros and M. R. D. Rodrigues, "Secrecy capacity of wireless channels," in Proceedings of IEEE International Symposium on Information Theory, Seattle, USA, Jul. 2006.

[7] Y. Liang, H. V. Poor, and S. Shamai (Shitz), "Secure communication over fading channels," IEEE Transactions on Information Theory, vol. 54, no. 6, pp. 2470-2492, Jun. 2008.

[8] P. K. Gopala, L. Lai, and H. E. Gamal, "On the secrecy capacity of fading channels," IEEE Transactions on Information Theory, vol. 54, no. 10, pp. 4687-4698, Oct. 2008.

[9] Y. Liang and H. V. Poor, "Generalized multiple access channels with confidential messages," in Proceedings of IEEE International Symposium on Information Theory, Seattle, USA, Jul. 2006.

[10] Y. Liang and H. V. Poor, "Multiple-access channels with confidential messages," IEEE Transactions on Information Theory, vol. 54, no. 3, pp. 976-1002, Mar. 2008.

[11] I. Csiszár and P. Narayan, "Secrecy capacities for multiterminal channel models," IEEE Transactions on Information Theory, vol. 54, no. 6, pp. 2437-2452, Jun. 2008.

[12] A. Khisti, A. Tchamkerten, and G. W. Wornell, "Secure broadcasting over fading channels," IEEE Transactions on Information Theory, vol. 54, no. 6, pp. 2453-2469, Jun. 2008.

[13] L. Dong, Z. Han, A. P. Petropulu, and H. V. Poor, "Secure wireless communications via cooperation," in Proceedings of 46th Annual Allerton Conference on Communication, Control, and Computing, UIUC, Illinois, USA, Sep. 2008.

[14] L. Dong, Z. Han, A. P. Petropulu, and H. V. Poor, "Amplify-and-forward based cooperation for secure wireless communications," in Proceedings of IEEE International Conference on Acoustics, Speech, and Signal Processing, Taipei, Taiwan, Apr. 2009.

[15] E. Tekin and A. Yener, "Achievable Rates for the General Gaussian Multiple AccessWire-Tap Channel with Collective Secrecy," in Proceedings of the 44th Annual Allerton Conference on Communication, Control, and Computing, Monticello, IL, Sep. 2006.

[16] E. Tekin and A. Yener, "The multiple access wire-tap channel: wireless secrecy and cooperative jamming," in Proceedings of the Information Theory and Applications Workshop, San Diego, CA, Jan. 2007.

[17] E. Tekin and A. Yener, "Achievable Rates for Two-Way Wire-Tap Channels," in Proceedings of the IEEE International Symposium on Information Theory, Nice, France, Jun. 2007.

[18] E. Ekrem and S. Ulukus, Cooperative Secrecy in Wireless Communications, in Securing Wireless Communications at the Physical Layer, W. Trappe and R. Liu, Eds., Springer-Verlag, 2009.

[19] E. Ekrem and S. Ulukus, "Secrecy in Cooperative Relay Broadcast Channels," IEEE Trans. on Information Theory, 57(1):137-155, Jan. 2011.

[20] X. He and A. Yener, "On the Equivocation Region of Relay Channels with Orthogonal Components," in Proceedings of the 41st Annual Asilomar Conference on Signals, Systems, and Computers, Pacific Grove, CA, Nov. 2007.

[21] X. He and A. Yener, "The Role of an Untrusted Relay in Secret Communication," in Proceedings of the IEEE International Symposium on Information Theory, Toronto, Canada, July 2008.

[22] X. He and A. Yener, "End-to-end Secure Multi-hop Communication with Untrusted Relays is Possible," in Proceedings of the 42nd Annual Asilomar Conference on Signals, Systems, and Computers,Pacific Grove, CA, Nov. 2008. 
[23] Y. Oohama, "Coding for relay channels with confidential messages," in Proceedings of IEEE Information Theory Workshop, Cairns, Australia, Sep. 2001.

[24] Y. Oohama, "Capacity theorems for relay channels with confidential messages," in Proceedings of IEEE International Symposium on Information Theory, Nice, France, Jun. 2007.

[25] B. Rankov and A. Wittneben, "Achievable rate regions for the two-way relay channel," in Proceedings of IEEE International Symposium on Information Theory, Seattle, USA, Jul. 2006.

[26] B. Rankov and A. Wittneben, "Spectral efficient protocols for half-duplex fading relay channels," IEEE Journal on Selected Areas in Communications, vol. 25, no. 2, pp. 379-389, Feb. 2007.

[27] C. Hausl and J. Hagenauer, "Iterative network and channel decoding for the two-way relay channel," in Proceedings of IEEE International Conference on Communications, Istanbul, Turkey, Jun. 2006.

[28] T. Cui, T. Ho, and J. Kliewer, "Memoryless relay strategies for two-way relay channels," IEEE Transactions on Communications, vol. 57, no. 10, pp. 3132-3143, Oct. 2009.

[29] X. He and A. Yener, "On the role of feedback in two-way secure communication," in Proceedings of the 42nd Annual Asilomar Conference on Signals, Systems, and Computers, Pacific Grove, CA, Nov. 2008.

[30] Y. Jing, "A relay selection scheme for two-way amplify-and-forward relay networks," in International Conference on Wireless Communications Signal Processing, Nov. 2009.

[31] I. Krikidis, J. Thompson, and S. Mclaughlin, "Relay selection for secure cooperative networks with jamming," IEEE Transactions on Wireless Communications, vol. 8, no. 10, pp. 5003-5011, Oct. 2009.

[32] M.S. Alam, K. Zarifi, S. Affes, and A. Ghrayeb, "Design and performance analysis of distributed relay selection techniques in wireless networks," in Biennial Symposium on Communications (QBSC), pp. 163-167, May 2010

[33] A. Bletsas, H. Shin, and M. Z. Win, "Cooperative communications with outage-optimal opportunistic relaying," IEEE Transactions on Wireless Communications, vol. 6, no. 9, pp. 3450-3460, Sept. 2007.

[34] T. S. Rappaport, Wireless communicaitons principles and practice, 2nd ed. Prentice Hall, 2002.

[35] L. Lai and H. E. Gamal, "The relay-eavesdropper channel: Cooperation for secrecy," IEEE Transactions on Information Theory, vol. 54, no. 9, pp. 4005-4019, Sep. 2008. 